\title{
Currents, Torques, and Polarization Factors in Magnetic Tunnel Junctions
}

\author{
J. C. Slonczewski ${ }^{1}$ \\ IBM Watson Research Center, Box 218, Yorktown Heights, \\ NY 10598 USA
}

\author{
Received by Phys. Rev. B on April 7, 2004. Revised on
}

September 20, 2018

\begin{abstract}
Bardeen's transfer-hamiltonian method is applied to magnetic tunnel junctions having a general degree of atomic disorder. The results reveal a close relationship between magneto-conduction and voltage-driven pseudo-torque, and also provide a means of predicting the thickness dependence of tunnelpolarization factors. Among the results: 1) The torque generally varies with moment direction as $\sin \theta$ at constant applied voltage. 2) Whenever polarization factors are well defined, the voltage-driven torque on each moment is uniquely proportional to the polarization factor of the other magnet. 3) At finite applied voltage, this relation implies significant voltage-asymmetry in the torque. For one sign of voltage the torque remains substantial even if the magnetoconductance is greatly diminished. 4) A broadly defined junction model, called ideal middle, allows for atomic disorder within the magnets and $\mathrm{F} / \mathrm{I}$ interface regions. In this model, the spin- $(\sigma)$ dependence of a basisstate weighting factor proportional to the sum over general state index $p$ of $\left(\iint d y d z \Psi_{p, \sigma}\right)^{2}$ evaluated within the (e.g. vacuum) barrier generalizes the local state density in previous theories of the tunnel-polarization factor. 5) For small applied voltage, tunnel-polarization factors remain legitimate up to first order in the inverse thickness of the ideal middle. An algebraic formula describes the first-order corrections to polarization factors in terms of newly defined lateral auto-correllation scales.
\end{abstract}

PACS: 85.75.-d 


\section{Introduction}

When first predicted, voltage-driven pseudo-torque in magnetic tunnel junctions (MTJs) appeared to be a marginal effect [1]. (Sec. 2 explains our use of the prefix pseudo- in the term pseudo-torque.) The lithographic scales and resistances available in early experimental MTJs appeared too large to permit anything more than a very small torque term in the Landau-Lifshitz equation. Resistive heating of the MTJ would have limited its possible consequences to only a small voltage-driven decrease of linewidth of narrowly-focussed Brillouin scattering. (This prediction was never tested.) As a result, one could not yet predict anything as remarkable as the now well-established magnetic reversal and high-frequency precession observed when the resistive barrier is replaced by a metallic spacer. For recent experimental work and earlier references dealing with switching and current-driven oscillations involving metallic spacers, see Refs. [2] and [3].

But in recent years, experimental activity in tunneling magnetoresistance has expanded vastly. It is fueled in great part by the experimental discovery of substantial tunneling magnetoresistance [4] at room-temperature and the resulting intensive exploration of non-volatile magnetic memory reviewed recently [5]. A part of this activity is the search for junction compositions and deposition techniques which lower the resistance to values more suitable for integrated-circuit application. Indeed, there now exist very recent experimental reports of current-driven switching in MTJs [6, 7]. This development may make possible two-terminal memory elements avoiding resort to three-terminal devices using both a metallic spacer for switching and a tunnel barrier for reading [8].

According to recent reviews of tunneling magneto-resistance [9, 10, 11, 12], empirical ferromagnet polarization coefficients $P_{i}[i=\mathrm{L}, \mathrm{R}$ refer to left and right magnets $\mathrm{F}_{i}$ in Fig. 1(a).] measured with $\mathrm{F}_{i} \mathrm{IS}$ junctions having a superconducting counter electrode [13] account well for magneto-resistance in FIF junctions. Let the formula

$$
J(V, \theta)=-J_{0}(V)[1+\iota(V) \cos \theta] \text {, with } J_{0}>0 \text { for } V>0
$$

for current density at constant applied voltage $V$ define the dimensionless coefficient $\iota$ of magneto-conduction. Here $\theta$ is the angle between the moments. (The - sign occurs in Eq. (1) because of the convention in Fig. 1 where particle-number current is positive for $V>0$.) In this article, the coefficient $\iota$ is more convenient than the experimentally preferred low-voltage tunneling-magnetoresistance ratio

$$
\mathrm{TMR}=\left(R_{\mathrm{AP}}-R_{\mathrm{P}}\right) / R_{\mathrm{P}}=2 \iota /(1-\iota) .
$$

\footnotetext{
${ }^{1}$ IBM RSM Emeritus.

john.slonczewski@verizon.net
} 
The original equation due to Julliere [14], expressed in our notation by the formula

$$
\iota=P_{\mathrm{L}} P_{\mathrm{R}}
$$

enjoys considerable success in interpreting experiments [9]. We find below that whenever $\iota$ separates this way into two polarization factors characteristic of the respective electrode-and-barrier compositions, pseudo-torque expressions having dimensionless coefficients $\tau_{\mathrm{L}}$ and $\tau_{\mathrm{R}}$ [See Eqs. (13), (19), and (20) below.], whose simplicity parallels that of Eqs.(1) and (3), hold also. The presence of the same average current density $J_{0}(V)$ in equations both for magneto-current and torque represents a strong connection between these two phenomena.

After the commonalities in Secs. 2 and 3, these mutual relations (Secs. 4 and 5) between magneto-conductance and pseudo-torques constitute the first of two parts of the present article. The second part (Secs. 6 and 7) is stimulated by the fact that theory does not generally support the separability of spin-channel currents into the left-dependent and right-dependent factors needed to justify polarization factors in the first place. Previous theories attack the question of polarization coefficients within the context of real electron structure by considering the transmission of electrons initially occupying well-defined crystalline-momentum states $[15,16,17]$. They posit either complete absence of disorder or special types of disorder only within the barrier to legitimize tunnel-polarization factors. The present approach, detailed below, complements those works by excluding disorder only from a subregion of the barrier.

Electron scattering, which causes metallic resistivity, abounds within experimental MTJ electrodes. A new feature of the present work is to forego altogether crystal-momentum quantization within the electrodes. This feature is particularly appropriate to contemporaneous experiments relying for electrodes on evaporated or sputtered magnetic elements and alloys having high defect concentration $[2,3,6,7]$. Both alloying and structural defects may cause an electron to scatter many times within the electrodes before and after it tunnels across the barrier so that initial and final crystal momenta are undefined.

Our elastic-tunneling theory rests on Bardeen's transfer-hamiltonian method (BTM) $[18,19]$, which is applicable to tunneling transitions between thermal baths of electron states without any spatially conserved observables. Bardeen defines two sets of basis states - one for the left electrode-and-barrier and one for the right electrode-andbarrier. Fermi's "golden rule" for transition rates gives the tunneling current. Thus our theory of MTJs has broader application than many others, previously reviewed $[9,11,12]$, which rely on scattering of Bloch electrons. Although more modern than 

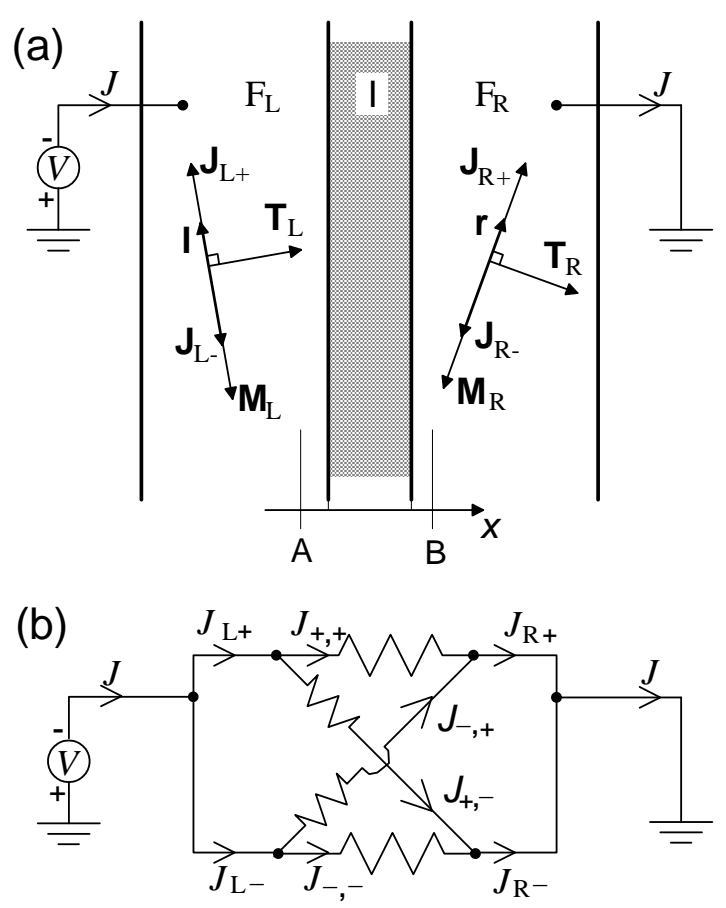

Figure 1: (a) Scheme of magnetic tunnel junction and key to notations. (b) Equivalent circuit for spin-channel currents and further key to notations.

Bardeen's method, they must assume defined initial and final momenta.

Our model of the junction, called ideal middle, excludes disorder only from a central geometric slab of uniform thickness $w$, which may consist of vacuum or periodic crystal lying somewhere within the barrier. We find that exact factorization of channel-to-channel current, which leads to Eqs.(3) and (19) below, occurs in the limit $w \rightarrow \infty$, just as in the case of complete absence of disorder. Further, our parametrization of lateral auto-corellation (See Sec. 7) of the Bardeen basisfunction sets predicts well-defined tunneling-polarization factors for finite barriers to first order in $w^{-1}$, which enhances their legitimacy for interpretation of experiments involving any degree of disorder. Computations and measurements of the new corellation-scale parameters $\xi_{\sigma}$ could shed quantitative light on the genesis of polarization factors.

By way of organization, Section 1 is this Introduction and Section 2 shows how 
spin-channel tunnel currents generally determine voltage-driven torque. Section 3 uses the BTM to derive the resulting fully general expressions for the magnetoconduction, the torques, and the relevant dimensionless coefficients $\iota, \tau_{\mathrm{L}}$, and $\tau_{\mathrm{R}}$. Section 4 shows how tunneling-polarization factors and the resulting simple expressions for $\iota, \tau_{\mathrm{L}}$, and $\tau_{\mathrm{R}}$ arise from a formal separability condition. Section 5 addresses the expressions for voltage-unsymmetric torque arising from voltage dependences of polarization factors. Section 6 demonstrates the separation condition and derives the tunnel-polarization factors which arise in the ideal-middle model at $w \rightarrow \infty$. Section 7 expands the magnetic tunneling properties for finite $w$ and derives a formula for the first-order $w^{-1}$-dependence of tunnel-polarization factors. Section 8 summarizes and discusses the results.

\section{First currents, then pseudo-torques}

Whenever two ferromagnets are separated by a nonmagnetic spacer, whether a tunneling barrier or a metal, exchange-generated pseudo-torques acting on the magnetic moments are attributable to the flow of spin-polarized current. For a fuller discussion of the genesis of pseudo(or effective)-torque from the principle of spin continuity, see Appendix B of Ref. [20]. Essential is the interpretation of magnetization dynamics $(\dot{\mathbf{M}} \equiv d \mathbf{M} / d t)$ governed by the additive terms in the macroscopic Landau-Lifshitz equation. Ordinarily $\dot{\mathbf{M}}$ represents the precession in place of electron-spin momentum localized to a volume element $d V$ due to local causes like magnetic field, spin-orbit coupling, etc. But the term describing externally driven spin transfer is transparently different. It reflects directly the flow of spin momentum directly into $d V$.

Indeed, the same may be said about the phenomenological exchange stiffness described commonly by the effective field $2 A \nabla^{2} \mathbf{m}$, with $\mathbf{M} \equiv M_{\mathrm{s}} \mathbf{m}$. This truth is masked by the derivability of ordinary exchange torque from variation of the stored energy

density $A \Sigma_{i, j}\left(\partial m_{i} / \partial x_{j}\right)^{2}$. Because spin transfer is driven by an externally supplied current or voltage, its effect cannot be derived from a stored energy. Therefore, its calculation requires direct recourse to spin currents as detailed below. Since this distinction between torque and divergence of polarization makes no difference in the subsequent application of the Landau-Lifshitz equation, the prefix "pseudo-" will be omitted in the remainder of this article.

Return now to our problem of spin-transfer torque created by external voltage applied to the MTJ. Consider particularly the series electric circuit in Fig. 1 (a) in which an external voltage $V$ causes electric-current density $J$ to flow in series through 
a left metallic ferromagnetic film $\mathrm{F}_{\mathrm{L}}$, a thin insulator I serving as a tunnel barrier, and finally a grounded right metallic ferromagnetic film $\mathrm{F}_{\mathrm{R}}$. By assumption, $\mathrm{F}_{\mathrm{L}}$ is sufficiently thin for the direction of spontaneous magnetization $\mathbf{M}_{\mathrm{L}}(x)=-M_{\mathrm{L}}(x) \mathbf{l}$ within $\mathrm{F}_{\mathrm{L}}$ not to depend on the plane-perpendicular coordinate $x$; similarly $\mathbf{M}_{\mathrm{R}}(x)=$ $-M_{\mathrm{R}}(x) \mathbf{r}$ within $\mathrm{F}_{\mathrm{R}}$. But the spontaneous magnetizations $M_{\mathrm{L}}$ and $M_{\mathrm{R}}$ may vary with $x$. (Here the three-dimensional unit vectors $\mathbf{l}$ and $\mathbf{r}$ include the angle $\theta=\cos ^{-1} \mathbf{r} \cdot \mathbf{l}$.) Thus we lay aside those possibilities of forward spin-wave excitation [21] and volumeintensive torque [22], arising from dependence of magnetization direction on $x$, which become significant for larger film thickness and current density.

One goal is to calculate the component $T_{\mathrm{R}}$ of interfacial torque vector $\mathbf{T}_{\mathrm{R}}$ per unit area, acting on $\mathbf{M}_{\mathrm{R}}$, which lies orthogonal to $\mathbf{r}$ within the instantaneous plane common to $\mathbf{l}$ and $\mathbf{r}$ as indicated in Fig. 1a. (The orientation of the magnetic space spanned by $\mathbf{l}$ or $\mathbf{r}$ is completely disconnected from that of position space $x, y, z$.) A general expression for $T_{\mathrm{R}}[20,23]$ reads thus:

$$
T_{\mathrm{R}}=\hbar\left[J_{\mathrm{L},+}-J_{\mathrm{L},-}+\left(J_{\mathrm{R},-}-J_{\mathrm{R},+}\right) \cos \theta\right] / 2 e \sin \theta
$$

Here the left spin-channel electric current densities $\mathbf{J}_{\mathrm{L}, \pm}=J_{\mathrm{L}, \pm} \mathbf{l}$ flow through plane A (See below) in direction $x$ and the right $\mathbf{J}_{\mathrm{R}, \pm}=J_{\mathrm{R}, \pm} \mathbf{r}$ flow through plane B. The factor $-\hbar / 2 e$ converts any electric channel current to one of spin momentum. A similar expression holds for the pseudo-torque $T_{\mathrm{L}}$ on the left moment. The torques $T_{\mathrm{R}}$ and $T_{\mathrm{L}}$ must generally be included in the dynamic Landau-Lifshitz equations for the two magnetic films.

Although previously applied only to all-metallic multilayers, Eq. (4) may also be used when the spacer is an insulator. For its derivation, one posits the nonrelativistic n-electron hamiltonian including, besides kinetic energy, coulomb terms due to external voltage and electron-nuclear and electron-electron interactions. In addition, one accepts the microscopically-based approximation, defensible in the case of Co, that the transverse (to local $\mathbf{M}$ ) components of conduction-electron spin polarization created at the two internal I/F interfaces decay to zero well within a characteristic distance $d_{\perp} \approx 1 \mathrm{~nm}$ [20], which was estimated explicitly for $\mathrm{Co} / \mathrm{Cu}$ and other interfacial compositions by scattering computations [24]. Moreover, in one experiment the threshold current for switching of Co by polarized current flowing through a metallic spacer is simply proportional to film thickness down to $1 \mathrm{~nm}$, confirming that the transverse polarization inside the ferromagnetic film vanishes at this scale [25]. Therefore the currents in the left and right magnets must be polarized along instantaneous left (l) and right (r) moment axes at depths greater than $d_{\perp}$ from the $\mathrm{F} / \mathrm{I}$ interfaces. Thus our work excludes thicknesses $<1 \mathrm{~nm}$, which require special treatment sensitive to atomic layering [26]. 
In the extensive literature on tunneling magnetoresistance involving $\mathrm{Fe}, \mathrm{Co}, \mathrm{Ni}$ and magnetically concentrated alloys of these elements with others of lower atomic number, there is little indication of spin relaxation at $\mathrm{I} / \mathrm{F}$ interfaces. Moreover experiments at cryogenic temperatures reveal that the distance $\lambda_{\|}$of spin relaxation due to spin-orbit coupling for the polarization component along the axis $\mathbf{M}$ is about $50 \mathrm{~nm}$ for Co and about $5.5 \mathrm{~nm}$ for Ni-Fe [27]. Thus it follows that, at least in the case of Co where $\lambda_{\|}>>d_{\perp}$, the channel currents $J_{\mathrm{L}, \pm}$ and $J_{\mathrm{R}, \pm}$ should be evaluated at the planes $\mathrm{A}$ and $\mathrm{B}$ lying at the distance $d_{\perp}$ from the respective $\mathrm{F} / \mathrm{I}$ interfaces. For within the space between these planes one may neglect spin-orbit effects and embrace the well-known spin-continuity relation which equates the sum of equivalent interfacial pseudo-torques with the net inflow of spin current [20, 23], having polarization directions $\mathbf{l}$ on the $\mathrm{L}$ side and $\mathbf{r}$ on the $\mathrm{R}$ side. In the notation of Fig. 1 (a), the statistical average of this equality becomes

$$
\mathbf{T}_{\mathrm{L}}+\mathbf{T}_{\mathrm{R}}=\frac{\hbar}{2 e}\left[\left(J_{\mathrm{L},-}-J_{\mathrm{L},+}\right) \mathbf{l}+\left(J_{\mathrm{R},+}-J_{\mathrm{R},-}\right) \mathbf{r}\right]
$$

By our assumed neglect of changes in $M_{\mathrm{L}}$, we write $\mathbf{l} \cdot \mathbf{T}_{\mathrm{L}}=0$. Therefore the scalar product of Eq. (5) with $\mathbf{l}$ eliminates $\mathbf{T}_{\mathrm{L}}$ and gives Eq. (4) for the magnitude $T_{\mathrm{R}}$. A similar equation holds for $T_{\mathrm{L}}$.

The above argument neglects a decaying and spatially oscillating transverse current, calculated in certain FNF cases to lie between 0 and $\simeq 10 \%$ of the incident spin current (See Fig. 7 of Ref. [24]). It is likely due to specular interference created at the perfect interface assumed in the calculation. Studies of FMF exchange coupling in vogue 10 years ago suggest that extremals in the Fermi surface determine the wavelength and cause the amplitude to decay with distance. The amplitude will be decreased by irregularities at real imperfect interfaces.

Even in the absence of applied electric voltage $(V=0)$ an additional perpendicular component of exchange pseudo-torque $\mathbf{T}_{\mathrm{R} \perp}=K \mathbf{l} \times \mathbf{r}=-\mathbf{T}_{\mathrm{L} \perp \text { predicted for MTJs }}$ [1] is generally related to phenomenological coupling energy $-K \mathbf{l} \bullet \mathbf{r}=-K \cos \theta$. It must also be included in the Landau-Lifshitz equation for the dynamics of magnet $\mathrm{F}_{\mathrm{R}}$. However, in that toy rectangular-barrier MTJ model [1], the (uncalculated) dependence of $\mathbf{T}_{\mathrm{R} \perp}$ and $\mathbf{T}_{\mathrm{L} \perp}$ on applied voltage occurred only in higher order $\left(\propto V^{2}\right)$ than the torque given by Eq. (4) $(\propto V)$. Moreover, its dynamic effect is relatively weaker in structures with coincident easy anisotropy axes and low magnetic damping, such as the pillars using metallic spacers experimentally favored for efficient currentdriven switching [2]. Indeed, steady oscillation excited by a steady electric current, such as that observed $[2,3]$, is possible with $\mathbf{T}_{\mathrm{R} \perp}=0$, but not in the absence of inplane $\mathbf{T}_{\mathrm{R}}$. In addition, the BTM used here does not readily provide this out-of-plane 
torque. For these reasons, we do not attempt to predict the perpendicular torque component in this work.

\section{Magneto-conduction and torques}

Equation (4) effectively reduces the interacting-electron problem of voltage-driven torque to the customarily independent-electron problem of spin-channel currents. One recently reviewed BTM-based theory of collinear MTJ magnetoresistance [11] extends naturally to tunneling between spin channels for general $\theta$. For adaptation of the BTM $[18,19]$ to the MTJ of Fig. 1a, a stationary basis state $|p, \sigma\rangle$ within the electron reservoir $\mathrm{F}_{\mathrm{L}}$ is assigned orbital index $p$ and majority/minority spin $\sigma= \pm$ quantized along axis $\mathbf{l}$. It satisfies $\left(H+e V-\epsilon_{p, \sigma}\right)|p, \sigma\rangle=0$, and decays exponentially within the barrier, considered semi-infinite in width when defining the basis states. Here, $H=p^{2} / 2 m+\Sigma_{\sigma}|\sigma\rangle U_{\sigma}(x, y, z)\langle\sigma|$, where the potential $U_{\sigma}$ depends on spin within the ferromagnets according to intinerant-electron magnetism theory [28], but not within the barrier. Within $\mathrm{F}_{\mathrm{R}}$, a similar state satisfies $\left(H-\epsilon_{q, \sigma^{\prime}}\right)\left|q, \sigma^{\prime}\right\rangle=0$ with quantization axis $\mathbf{r}$. Because the barrier is assumed to dominate all other resistances of this circuit, the spin channels are shown in Fig. 1 (b) as shorted in each magnet and/or external-contact region by spin lattice relaxation due to spin-orbit coupling. One may disregard spin accumulation and the related distinction between electric and electrochemical potentials which are important when a non-magnetic metallic spacer substitutes for the barrier [29]. $\quad U_{\sigma}$ includes all elastic terms arising from atomic disorder due to alloying, defects, interfacial atomic interdiffusion, etc. The state indices $p, q$ simply enumerate the exact eigenstates $|p, \sigma\rangle,\left|q, \sigma^{\prime}\right\rangle$ of $H$ in the Bardeen basis. Each such state incorporates effects of all multiple elastic scatterings without limit.

Employing the spinor transformation connecting quantization axes $\mathbf{l}$ and $\mathbf{r}$, the transfer matrix element takes the form

$$
\left\langle p, \sigma|H-\varepsilon| q, \sigma^{\prime}\right\rangle=\left[\begin{array}{cc}
\gamma_{p,+; q,+} \cos \frac{\theta}{2} & \gamma_{p,+; q,-} \sin \frac{\theta}{2} \\
-\gamma_{p,-; q,+} \sin \frac{\theta}{2} & \gamma_{p,-; q,-} \cos \frac{\theta}{2}
\end{array}\right] .
$$

Direct extension of BTM [30] to our spin-dependent case gives the expression

$$
\gamma_{p, \sigma ; q, \sigma^{\prime}}(x)=\frac{-\hbar^{2}}{2 m} \int d y d z\left(\psi_{p, \sigma} \partial_{x} \varphi_{q, \sigma^{\prime}}-\varphi_{q, \sigma^{\prime}} \partial_{x} \psi_{p, \sigma}\right)
$$

where the integral is over unit area for coordinate $x$ lying appropriately (see below) inside the barrier. The energies $\epsilon_{p, \sigma}$ and $\epsilon_{q, \sigma^{\prime}}$ may differ only infinitesimally from 
the Fermi value $\varepsilon=\varepsilon_{\mathrm{F}}$. The hamiltonian $H$, the left $\left(\psi_{p, \sigma}\right)$ and right $\left(\varphi_{q, \sigma^{\prime}}\right)$ orbital wave functions, and these matrix elements (7) are real.

Only the neglect of cross-barrier overlaps $\left\langle p, \sigma \mid q, \sigma^{\prime}\right\rangle$ allows use of the Fermi golden rule of perturbation theory which is strictly valid for an orthonormal basis. Substitution of the perturbation (6) into this rule is followed by summation over the initial states in an infinitessimal energy band of width $\mathrm{eV}$. Thus the partial electric current density flowing between channel $\sigma$ in $\mathrm{F}_{\mathrm{L}}$ and channel $\sigma^{\prime}$ in $\mathrm{F}_{\mathrm{R}}$ becomes

$$
J_{\sigma, \sigma^{\prime}}=\frac{-2 \pi e^{2} V}{\hbar} \sum_{p, q}^{\prime}\left\langle p, \sigma\left|H-\varepsilon_{\mathrm{F}}\right| q, \sigma^{\prime}\right\rangle^{2}
$$

at $T=0 \mathrm{~K}$. The ${ }^{\prime}$ in $\sum_{p, q}^{\prime}$ imposes the conditions $\varepsilon_{\mathrm{F}}<\left(\varepsilon_{p, \sigma}, \varepsilon_{q, \sigma^{\prime}}\right)<\varepsilon_{\mathrm{F}}+e V$.

Notations in the equivalent circuit shown in Fig. 1 (b) make plain the relations

$$
J_{\mathrm{L} \sigma}=J_{\sigma,+}+J_{\sigma,-}, \quad J_{\mathrm{R} \sigma^{\prime}}=J_{+, \sigma^{\prime}}+J_{-, \sigma^{\prime}}, \quad\left(\sigma, \sigma^{\prime}= \pm\right)
$$

needed in Eq. (4). The right hand sides of these equations are evaluated from Eqs. (6-8).

Next we write the total electric current density $J=J_{\mathrm{L},+}+J_{\mathrm{L},-}$. With the notation

$$
\Gamma_{\sigma, \sigma^{\prime}}=\frac{2 \pi e V}{\hbar} \sum_{p, q}^{\prime} \gamma_{p, \sigma ; q, \sigma^{\prime}}^{2}
$$

for interchannel particle-number tunneling conduction with the angular factor omitted, the above equations combine to give Eq. (1) with

$$
J_{0}=e\left(\Gamma_{+,+}+\Gamma_{-,-}+\Gamma_{+,-}+\Gamma_{-,+}\right) / 2
$$

and the electric magneto-conduction coefficient

$$
\iota=e\left(\Gamma_{+,+}+\Gamma_{-,-}-\Gamma_{+,-}-\Gamma_{-,+}\right) / 2 J_{0} .
$$

Eq.(4) becomes

$$
T_{\mathrm{R}}=-\left(\hbar \tau_{\mathrm{R}} J_{0} / 2 e\right) \sin \theta
$$

or, in coordinate-free form

$$
\mathbf{T}_{\mathrm{R}}=\left(\hbar \tau_{\mathrm{R}} J_{0} / 2 e\right) \mathbf{r} \times(\mathbf{l} \times \mathbf{r}),
$$

with the torque coefficient

$$
\tau_{\mathrm{R}}=e\left(\Gamma_{+,+}+\Gamma_{+,-}-\Gamma_{-,-}-\Gamma_{-,+}\right) / 2 J_{0} .
$$

The fact that the linear combination of the parameters $\Gamma_{\sigma, \sigma^{\prime}}$ appearing in Eq. (12) differs from that in Eq. (15) and a similar one for $\mathbf{T}_{\mathrm{L}}$ precludes any fully general connection between torques and electrical current. 


\section{Left-right separability and polarization factors}

Particularly interesting relations arise if the summation in Eq. (10) for the interchannel particle current happens to separate into left- and right-dependent factors in the form

$$
\Gamma_{\sigma, \sigma^{\prime}}=f \Omega_{\mathrm{L}, \sigma} \Omega_{\mathrm{R}, \sigma^{\prime}} .
$$

Here the coefficient $f$, which we make no attempt to evaluate, is independent of $\sigma, \sigma^{\prime}$. (Sections 6 and 7 address conditions for this separability.) For then Eq. (11) gives

$$
J_{0}=\frac{e f}{2}\left(\Omega_{\mathrm{L},+}+\Omega_{\mathrm{L},-}\right)\left(\Omega_{\mathrm{R},+}+\Omega_{\mathrm{R},-}\right)
$$

and Eq. (12) gives Eq. (3) with the tunneling polarization parameters

$$
P_{i}=\frac{\Omega_{i,+}-\Omega_{i,-}}{\Omega_{i,+}+\Omega_{i,-}} \quad(i=\mathrm{L}, \mathrm{R})
$$

which are directly measurable using FIS junctions [9]. In these terms, Eqs. (1) and (3) give the magneto-conduction and Eq. (14) the torque with

$$
\tau_{\mathrm{R}}=P_{\mathrm{L}} .
$$

Similarly, the torque on the left magnet is

$$
T_{\mathrm{L}}=-\left(\hbar \tau_{\mathrm{L}} J_{0} / 2 e\right) \sin \theta, \quad \tau_{\mathrm{L}}=P_{\mathrm{R}}
$$

or, in coordinate-free form

$$
\mathbf{T}_{\mathrm{L}}=\frac{\hbar \tau_{\mathrm{L}}}{2 e} J_{0} \mathbf{l} \times(\mathbf{r} \times \mathbf{l}) .
$$

The Eqs.(3), (19), and (21) show the very close relation between current-driven torques and magneto-conduction at the same voltage, summarized by $\iota=\tau_{\mathrm{L}} \tau_{\mathrm{R}}$, if the separability condition (16) is satisfied.

The ground-breaking paper of Julliere [14] gave equations equivalent to (3) and (18) taking $\Omega_{\mathrm{L} \sigma}$ and $\Omega_{\mathrm{R} \sigma^{\prime}}$ to be spin-dependent basis-state densities at $\varepsilon=\varepsilon_{\mathrm{F}}$. It appeared to attribute the dimensionless magneto-current coefficient $\iota=P_{\mathrm{L}} P_{\mathrm{R}}$ to bulk properties of the two magnetic compositions involved. But the analytically solved free-electron rectangular-potential model [1] shows that an interface-dependent factor must be included in $\Omega_{i, \sigma}$ as well. The transfer-hamiltonian treatment of this toy model follows immediately from the spinless treatment [30] giving

$$
\Omega_{i, \sigma}=k_{i, \sigma} /\left(\kappa_{0}^{2}+k_{i, \sigma}^{2}\right)
$$


where

$$
k_{i, \sigma}^{2}=2 m E_{i, \sigma} / \hbar^{2} \text { and } \kappa_{0}^{2}=2 m B / \hbar^{2} .
$$

Here, $E_{i, \sigma}$ is the kinetic energy at the Fermi level and $B$ is the barrier potential measured from the Fermi level. Equation (18) now gives

$$
P_{i}=\frac{k_{i,+}-k_{i,-}}{k_{i,+}+k_{i,-}} \cdot \frac{\kappa_{0}^{2}-k_{i,+} k_{i,-}}{\kappa_{0}^{2}+k_{i,+} k_{i,-}}
$$

in agreement with Ref. [1]. In this formula, the first factor depends purely on basisstate densities in the magnet, while the second mixes magnet and barrier properties. The results of the toy model [1] satisfy the general magneto-conduction relations (1), (3) and torque relations $(14),(19),(21)$ with this substitution.

We note in passing that experimental variation of barrier height $B$ shows considerable support for the zero of $\iota$ at the barrier potential satisfying $\kappa_{0}^{2}-k_{i,+} k_{i,-}=0$ expected from Eq. (24) [31] (for small $V$ ). Therefore, in spite of its fundamental naivete, this toy model enjoys some degree of credibility. It illustrates the general fact that, even when separability holds, each polarization factor is a property of the electron structure of the magnet and barrier combination as demonstrated by many experiments and calculations. Section 7 will discuss how tunnel polarization may vary with barrier thickness.

\section{$5 \quad$ Finite bias and torque asymmetry}

In experiments, TMR typically decreases significantly with increasing finite $V[9]$. Voltage-dependence of interfacial transmission, special state density distributions, extrinsic impurity effects, and inelastic tunneling contribute to this decrease $[9,12]$. This is important because large voltages will be required to read and write in a 2-terminal memory element.

The toy polarizations of Eq. (24) will serve to illustrate qualitatively the very unsymmetric effect of finite $V$ on voltage-driven pseudo-torque. One calculation of TMR uses the WKB approximation for the free-electron wave function within the constant-slope barrier potential sketched in Fig. 2 [32]. The interfacial transmissions are approximated by those of the flat-potential polarizations (24). The authors cite some experimental support for their results.

It is the decrease of $P_{i}$ in the particular electrode which collects the tunneled electrons that primarily accounts for the decrease of $\iota$ in the calculated result [32]. In Fig. 2, for $V>0$, the collecting electrode lies on the right. Note that the electrons whose energy lies in a narrow band (shaded in Fig. 2) just below the Fermi level 
of the emitting electrode on the left of the barrier dominate the tunneling current because of the strong energy dependence of the WKB factor $\exp \left[-2 \int \kappa(x) d x\right]$ in the transmission coefficient. Since these hot electrons lie an amount well above the Fermi level on the right, this energy shift $\mathrm{eV}$ must be taken into account when estimating $P_{\mathrm{R}}$.

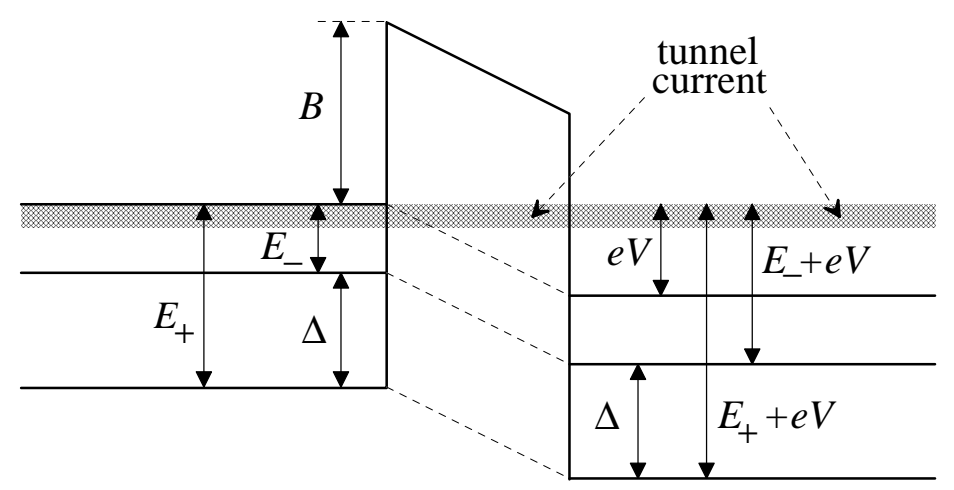

Figure 2: Schematic junction potential for finite V. The shaded bar indicates the energy range of most of the tunneling electrons.

We simplify this model one step further and neglect the width of the shaded current band in Fig. 2. It is then clear that Eqs. (23) and (24) with $i=\mathrm{L}$ are still correct for $P_{\mathrm{L}}$, neglecting correction for the finite slope of the barrier potential. However, the equations

$$
k_{\mathrm{R} \sigma}^{2}=2 m\left(E_{\mathrm{R} \sigma}+e V\right) / \hbar^{2} \text { and } \kappa_{0}^{2}=2 m(B-e V) / \hbar^{2},
$$

obtained by adding $\mathrm{eV}$ to each electron energy on the right, must replace Eqs.(23) for $i=\mathrm{R}$.

Figure 3 plots the curves $\tau_{\mathrm{L}}=P_{\mathrm{R}}$ and $\tau_{\mathrm{R}}=P_{\mathrm{L}}$ evaluated from the preceding three equations as well as TMR from Eqs. (2) and (3) versus $V$ for the special example of a symmetric junction with the parameters $k_{\mathrm{L}-}=k_{\mathrm{R}-} \equiv k_{-}, k_{\mathrm{L}+}=k_{\mathrm{R}+} \equiv 10 k_{-}$, and $\kappa_{0}=6.4 k_{-}$, whereby each electrode has the $V=0$ polarization $P_{\mathrm{L}}=P_{\mathrm{R}}=0.5$. In this illustration, $\operatorname{TMR}(V)$ is symmetric because it involves both $P_{\mathrm{L}}$ and $P_{\mathrm{R}}$ but $P_{\mathrm{L}, \mathrm{R}}(V)$ 
and the torque coefficients $\tau_{\mathrm{L}, \mathrm{R}}(V)$ are not. Although the theory preceding this section assumed small $V$, the present discussion makes reasonable the application of the results to finite $V$ with the understanding that the polarization of the collecting electrode generally depends more strongly on $V$. Of course, this toy calculation cannot make quantitative predictions of the $V$-dependence which must rest on details of electron structure $[9,12]$.

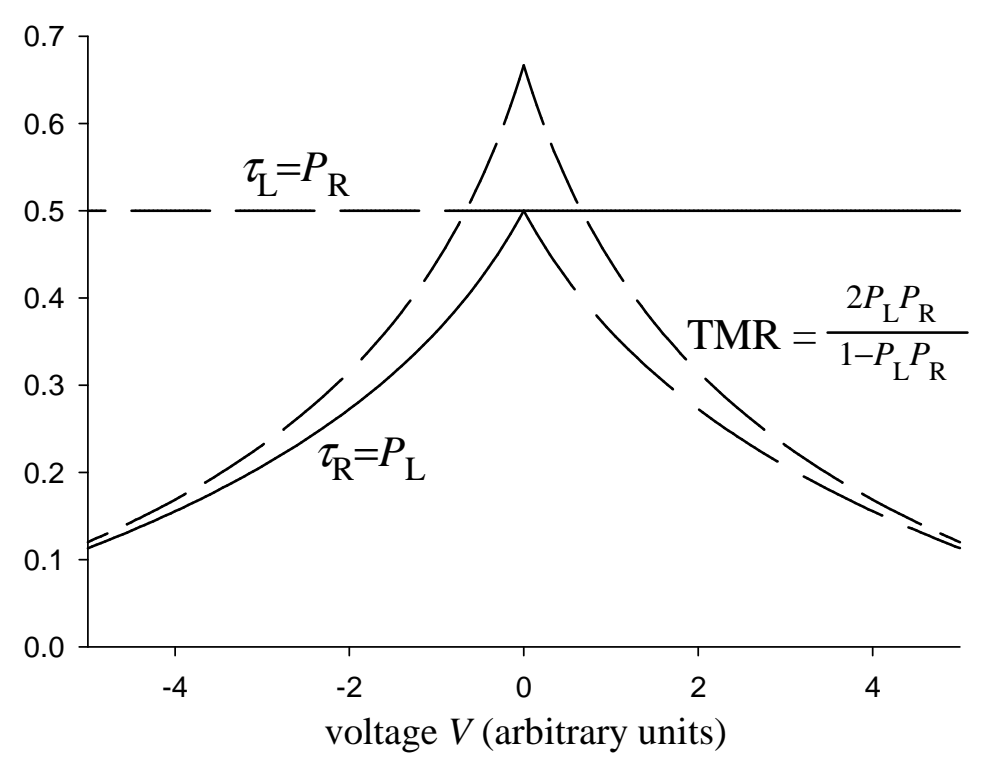

Figure 3: Schematic effect of finite voltage on TMR, polarization, and torque coefficients illustrated by the toy free electron model of a physically symmetric magnetic tunnel junction. Note that TMR is symmetric but the other coefficients are not. The parameters are $\kappa_{0}=6.4 k_{-}, k_{+}=10 k_{-}$.

Note that while critical current density for magnetic excitation is appropriate to junctions with metallic spacers, the high resistance of a MTJ makes critical voltage more appropriate. (Indeed, strictly speaking, the critical current of a constantcurrent generator will generally differ from the current density flowing at threshold in the presence of constant external voltage.) Another significant difference between metallic and insulating spacers lies in the angular symmetry of the torque. The fixed $\sin \theta$-dependence at constant $V$ in the tunneling case has no counterpart in the metallic case where more general torque expressions typically contribute to asym- 
metry of excitation threshold [20]. Now we see that the non-ohmic resistance of a tunneling barrier gives rise to the torque asymmetry of $\tau_{\mathrm{R}}(V)$ exhibited in Fig. 3, which naturally reflects in yet another origin for asymmetry of voltage threshold.

\section{Ideal-middle model for separability}

A recent publication compares existing theoretical arguments supporting the existence of tunnel-polarization factors [17]. Each of them assumes incident states with definite crystalline momentum. One common type of argument assumes complete absence of disorder so that the tunneling through a thick barrier is dominated by a single value of lateral momentum. A different model of Tsymbal and Pettifor [15] recovers factorization and therefore the Julliere formula in a tight-binding singleband model disordered only within the barrier. Similarly, the model of Mathon and Umerski attributes the factorization to phase decoherence due to disorder within the barrier $[9,16]$. These treatments are augmented with arguments based on the Feynman path integral in a disordered barrier [17]. Our treatment below complements these arguments with the contrary tack of foregoing lateral momentum quantization completely within the electrodes and I/F interfaces while preserving ideal crystalline ordering or vacuum within the middle of the barrier.

Figure 4 indicates the structural scheme. The left $\left(\psi_{p, \sigma}\right)$ and right $\left(\varphi_{q, \sigma^{\prime}}\right)$ orbital basis functions for the transfer matrix, introduced in Sec. 3, are governed in detail by the general potential $U_{\sigma \text { or } \sigma^{\prime}}$ depending on crystal structure, alloy composition, defects, F/I interface roughness and atomic interdiffusion, etc. The quantum numbers $p$ and $q$ do not refer to any diagonal operator. Exceptionally, the ideal-middle $\mathcal{B}$ of the barrier consists of an ideal crystalline slab or vacuum region defined by $a \leq x \leq b$ where the planes $x=a, b$ are dubbed portals of the ideal middle. In order to define the left and right basis-state sets of the Bardeen theory, the barrier potential extends into alternative semi-infinite spaces $(a \leq x)$ and $(x \leq b)$, where it is greater than $\varepsilon_{\mathrm{F}}$, independent of or periodically dependent on $y, z$ and independent of $\sigma$ and $\sigma^{\prime}$. The respective conditions $\psi_{p, \sigma} \rightarrow 0$ for $x \rightarrow \infty$ and $\varphi_{q, \sigma^{\prime}} \rightarrow 0$ for $x \rightarrow-\infty$ complete the definitions of $\psi_{p, \sigma}$ and $\varphi_{q, \sigma^{\prime}}$.

The effective-mass theorem [33] is valid when $\varepsilon$ is near the bottom $\mathbf{k}=\mathbf{k}_{0}$ of the conduction band within region $\mathcal{B}$. Then the evanescent portion of a left-magnet basis function within this region is approximated by

$$
\psi_{p, \sigma}=\Psi_{p, \sigma}(x, y, z) u_{\mathrm{cb}, \mathbf{k}_{0}}(x, y, z)
$$

where $\Psi_{p, \sigma}$ satisfies $\left(\mathcal{H}_{\mathrm{bar}}-\varepsilon_{p, \sigma}\right) \Psi_{p, \sigma}=0$ and $\Psi_{p, \sigma} \rightarrow 0$ for $x \rightarrow \infty$, and $u_{\mathrm{cb}, \mathbf{k}_{0}}$ is the Bloch function for the bottom of the conduction band. The effective barrier 


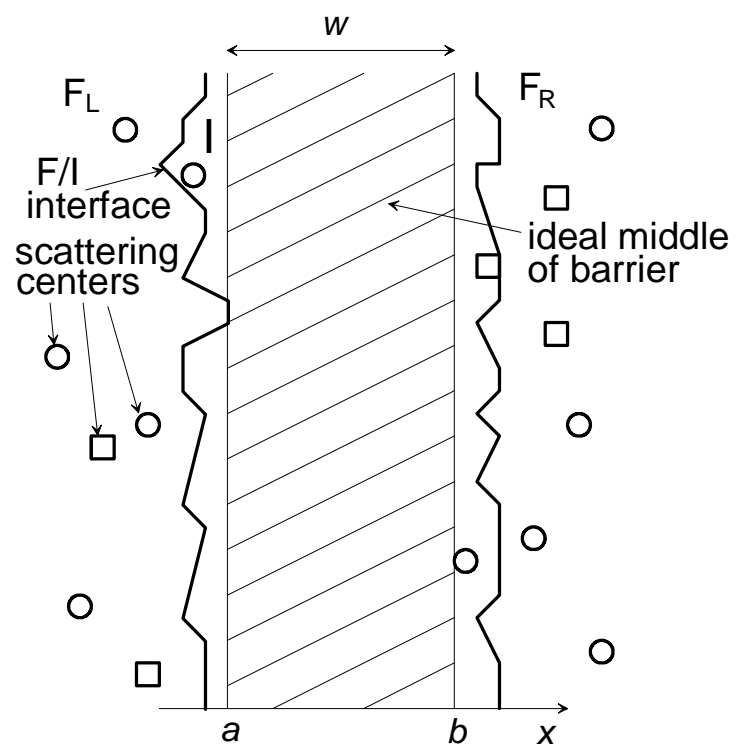

Figure 4: Depiction of the ideal-middle model of a magnetic tunneling junction. Disorder without limit is permitted in both electrodes and barrier except within a central slab $\mathcal{B}$ of the barrier lying between the portal planes $x=a, b$.

hamiltonian is $\mathcal{H}_{\mathrm{bar}}=-\hbar^{2} \nabla^{2} / 2 m_{\mathrm{cb}}+\mathcal{U}(x)$ where $m_{\mathrm{cb}}$ is the effective mass and $\mathcal{U}(x)\left(>\varepsilon_{\mathrm{F}}\right)$ is the spin-independent atomically smoothed effective barrier potential. Similarly for $\mathrm{F}_{\mathrm{R}}, \varphi_{q, \sigma^{\prime}}=\Phi_{q, \sigma^{\prime}} u_{\mathrm{cb}, \mathbf{k}_{0}}$ with $\Phi_{q, \sigma^{\prime}} \rightarrow 0$ for $x \rightarrow-\infty$. In case of vacuum, $(\Psi, \Phi)$ are indistinguishable from $(\psi, \varphi)$. (Note however that this treatment fails if both $V$ is finite and the FI interfaces are disordered, for then $\mathcal{U}$ depends on $y$ and $z$ as well as $x$.)

Assuming periodic boundary conditions in the $\mathbf{s}=(y, z)$ sub-space, the evanescent portions of left and right basis states within $\mathcal{B}$ are conveniently fourier-expanded in space $\mathbf{s}$ with the WKB approximation giving

$$
\Psi_{p, \sigma}=\sum_{\mathbf{k}} \lambda_{p, \sigma}(\mathbf{k})[\kappa(k, a) / \kappa(k, x)]^{1 / 2} \exp \left[-\int_{a}^{x} \kappa\left(k, x^{\prime}\right) d x^{\prime}+i \mathbf{k} \bullet \mathbf{s}\right]
$$

and

$$
\Phi_{q, \sigma^{\prime}}=\sum_{\mathbf{k}} \mu_{q, \sigma^{\prime}}(\mathbf{k})[\kappa(k, b) / \kappa(k, x)]^{1 / 2} \exp \left[-\int_{x}^{b} \kappa\left(k, x^{\prime}\right) d x^{\prime}+i \mathbf{k} \bullet \mathbf{s}\right]
$$

where the sums $\Sigma_{\mathbf{k}}$ are carried over a 2-dimensional reduced Brillouin zone. These formulas employ the function 


$$
\kappa(k, x)=\left[\kappa_{0}^{2}(x)+k^{2}\right]^{1 / 2}, \quad \text { with } \kappa_{0}^{2}=2 m_{\mathrm{cb}}\left[\mathcal{U}(x)-\varepsilon_{\mathrm{F}}\right] / \hbar^{2},
$$

where $i \kappa$ is the imaginary component of the wave-vector in region $\mathcal{B}$. Note that Eqs. (27) and (28) reduce to expansions of $\psi_{p, \sigma}$ and $\varphi_{q, \sigma^{\prime}}$ with coefficients $\lambda_{p, \sigma}(\mathbf{k})$ and $\mu_{q, \sigma^{\prime}}(\mathbf{k})$ on the portal planes $x=a$ and $x=b$ respectively.

The transfer-hamiltonian matrix element of Eq. (7) is evaluated at any $x$ lying within the interval $a \leq x \leq b$. Consequently $\Psi, \Phi$, and $m_{\mathrm{cb}}$ may replace $\psi, \varphi$, and $m$ respectively in this formula. One convenient choice to evaluate Eq. (7) is $x=x_{\max }$, satisfying $\mathcal{U}(x) \leq U\left(x_{\max }\right)$ for all $x$, because the resulting condition $\partial \kappa_{0} / \partial x\left(x_{\max }\right)=0$ simplifies the mathematics. (Inclusion in $\mathcal{U}$ of the image potential due to electron-electron correlation will often insure the presence of a maximum, even if $|V|$ is large.) Substitution of Eqs. (27) and(28) followed by integration over $y$ and $z$, with the assistance of the identity $\int d \mathbf{s}^{2} \exp \left[i\left(\mathbf{k}-\mathbf{k}^{\prime}\right) \cdot \mathbf{s}\right]=\delta_{\mathbf{k}, \mathbf{k}^{\prime}}$, reduces Eq. (7) to

$$
\gamma_{p, \sigma ; q, \sigma^{\prime}}=\Sigma_{\mathbf{k}} F(w, \mathbf{k}) \lambda_{p, \sigma}^{*}(\mathbf{k}) \mu_{q, \sigma^{\prime}}(\mathbf{k})
$$

where

$$
F(w, \mathbf{k})=\frac{-4 \pi^{2} \hbar^{2}}{m_{\mathrm{cb}}} \kappa^{1 / 2}(\mathbf{k}, a) \kappa^{1 / 2}(\mathbf{k}, b) \exp \left[-\int_{a}^{b} d x \kappa(k, x)\right] .
$$

Here we use the barrier-middle thickness $w=b-a$, and note $\lambda_{p, \sigma}^{*}(\mathbf{k})=\lambda_{p, \sigma}(-\mathbf{k})$ and $\mu_{q, \sigma^{\prime}}^{*}(\mathbf{k})=\mu_{q, \sigma^{\prime}}(-\mathbf{k})$ because $\Psi_{p, \sigma}$ and $\Phi_{q, \sigma^{\prime}}$ are real. [When $w$ varies in our discussion below, $\lambda_{p, \sigma}(\mathbf{k})$ and $\mu_{q, \sigma^{\prime}}(\mathbf{k})$ remain unchanged because they pertain to the semi-infinite barrier independent of $w$. We merely expand or contract the ideal middle of the barrier in Eq. (31).] After rearranging the order of sums, Eq. (10) with substitution of (30) and (31) becomes

$$
\Gamma_{\sigma, \sigma^{\prime}}=\frac{2 \pi e V}{\hbar} \sum_{\mathbf{k}} F(w, \mathbf{k}) \sum_{\mathbf{k}^{\prime}} F\left(w, \mathbf{k}^{\prime}\right) \mathcal{L}_{\sigma}\left(\mathbf{k}, \mathbf{k}^{\prime}\right) \mathcal{M}_{\sigma^{\prime}}\left(\mathbf{k}, \mathbf{k}^{\prime}\right)
$$

where each of the two functions

$$
\mathcal{L}_{\sigma}=\Sigma_{p}^{\prime} \lambda_{p, \sigma}^{*}(\mathbf{k}) \lambda_{p, \sigma}\left(\mathbf{k}^{\prime}\right), \mathcal{M}_{\sigma^{\prime}}=\Sigma_{q}^{\prime} \mu_{q, \sigma^{\prime}}(\mathbf{k}) \mu_{q, \sigma^{\prime}}^{*}\left(\mathbf{k}^{\prime}\right)
$$

depends only on parameters of the left and right magnet-and-barrier combinations respectively. The' on $\Sigma^{\prime}$ signifies the conditions given previously for Eq. (8).

In the presence of atomic disorder, the sums in Eqs. (33) are carried over many states of randomized character. Therefore they have the nature of statistical autocorrelations in $y, z$-space which should depend smoothly on $\mathbf{k}$ and $\mathbf{k}^{\prime}$ and are Taylorexpandable about $\mathbf{k}=\mathbf{k}^{\prime}=0$. (See Sec. 5 for the very different toy free-electron case 
of vanishing disorder [1], in which one may formally replace $p \rightarrow \mathbf{k}^{\prime \prime}, q \rightarrow \mathbf{k}^{\prime \prime \prime}$ so that $\mathcal{L}_{\sigma}$ and $\mathcal{M}_{\sigma^{\prime}}$ become proportional to $\delta_{\mathbf{k}, \mathbf{k}^{\prime}}$ ) In addition, with increasing thickness $w=b-a$ of region $\mathcal{B}$, the exponential in Eq.(31) becomes ever more sharply peaked at $\mathbf{k}=0$. Summation over $\mathbf{k}$ and $\mathbf{k}^{\prime}$ of the terms in these Taylor series' for finite $w$ gives the corresponding terms

$$
\Gamma_{\sigma, \sigma^{\prime}}(w)=\Gamma_{\sigma, \sigma^{\prime}}^{(0)}(w)+\Gamma_{\sigma, \sigma^{\prime}}^{(1)}(w)+\cdots
$$

The initial constants in both Taylor expansions yield

$$
\Gamma_{\sigma, \sigma^{\prime}}^{(0)}(w)=f(w) \Omega_{\mathrm{L}, \sigma}^{(0)} \Omega_{\mathrm{R}, \sigma}^{(0)}
$$

with $\Omega_{\mathrm{L}, \sigma}^{(0)} \equiv \mathcal{L}_{\sigma}(0,0)$ and $\Omega_{\mathrm{R}, \sigma^{\prime}}^{(0)} \equiv \mathcal{M}_{\sigma^{\prime}}(0,0)$. Here factors independent of $\sigma$ and $\sigma^{\prime}$ are absorbed into $f$. Therefore, to leading order in this expansion, the integrations in Eq. (32) tend to the left-right separation of the form (16).

Written in full, the parameters needed in the general polarization formula (18) are, to lowest order in the Taylor expansions of Eqs. (33), the basis-state weights

$$
\begin{aligned}
\Omega_{\mathrm{L}, \sigma}^{(0)} & =\sum_{p}\left(\iint d y d z \Psi_{p, \sigma}(a, y, z)\right)^{2} \\
\Omega_{\mathrm{R}, \sigma^{\prime}}^{(0)} & =\sum_{q}\left(\iint d y d z \Phi_{q, \sigma^{\prime}}(b, y, z)\right)^{2}
\end{aligned}
$$

where $\iint d y d z$ is carried over unit junction area at the portal positions $a$ and $b$. [See the next section for development of $\left.\Gamma_{\sigma, \sigma^{\prime}}^{(1)}(w).\right]$ Note that the latter two equations differ generally from the local state (or charge) density often cited in connection with tunneling. (LSD $\left.\propto \iint d y d z \Psi_{p, \sigma}^{2}\right)$ They reduce to the LSD in the complete absence of disorder when each of the two sums reduces to a single term $\Psi_{\mathbf{k}=0, \sigma}^{2}$ and $\Phi_{\mathbf{k}=0, \sigma^{\prime}}^{2}$ independent of $y$ and $z$.

\section{Correction of polarization at finite thickness}

The non-orthogonality between left and right basis functions constitutes a basic weakness of the BTM. Even though the validity of golden-rule transition rates in BTM is not generally assured, it has an enormous acceptance in the literature. The toy free-electron MTJ theory, though founded directly on a solution of the wave equation in the entire ideal non-disordered FIF system having a flat barrier potential, was evaluated only to leading order in the exponential parameter $e^{-\kappa w}[1]$. The BTM 
calculation for the same model agrees exactly with its results, as one knew it should from previous non-spin dependent tunneling theory [19].

Let us assume that BTM is correct to the same exponential degree for our idealmiddle model as for the toy model. The previous section showed that the BTM supports tunnel-polarization phenomenology in lowest order. Continuing with BTM, we derive here a correction to the formulas (18), (36), and (37) for polarization which we find below varies algebraically, not exponentially, with $w^{-1}$. Therefore these corrections should be reliable in spite of this general weakness of the BTM.

Further progress requires parametrization of the autocorellation functions defined by Eq. (33). Note first the consequence of assuming that the possibly disordered atomic configuration in $\mathrm{F}_{\mathrm{L}}$ produces no electrostatic potential in $\mathrm{F}_{\mathrm{R}}$ and vice versa. From Eqs. (27), (28), and (33), in-plane translation of the (disordered) microscopic potential of only the left electrode according to $\mathbf{s} \rightarrow \mathbf{s}+(B, C)$, where $(B, C)$ is a periodic-lattice translation of the barrier middle, has the effects, from Eq. (33), $\mathcal{L}_{\sigma} \rightarrow \mathcal{L}_{\sigma} \exp \left[i\left(\mathbf{k}^{\prime}-\mathbf{k}\right) \cdot(B, C)\right]$ and $\mathcal{M}_{\sigma^{\prime}} \rightarrow \mathcal{M}_{\sigma^{\prime}}$. Averaging over all possible such phase changes makes $\mathcal{L}_{\sigma}$ and $\mathcal{M}_{\sigma^{\prime}}$ diagonal and eliminates all terms with $\mathbf{k} \neq \mathbf{k}^{\prime}$ from the double sum in Eq. (32). This equation now becomes

$$
\Gamma_{\sigma, \sigma^{\prime}}=\frac{2 \pi e V}{\hbar} \sum_{\mathbf{k}} F^{2}(\mathbf{k}) \mathcal{L}_{\sigma}(\mathbf{k}) \mathcal{M}_{\sigma^{\prime}}(\mathbf{k})
$$

using the now diagonal forms of $\mathcal{L}_{\sigma}$ and $\mathcal{M}_{\sigma^{\prime}}$.

Parenthetically, note that in the special case of vanishing disorder, the state indices $p$ and $q$ become $m, \mathbf{k}$ and $n$, $\mathbf{k}$ respectively, with $m, n$ the respective band indices and $\mathbf{k}$ the lateral crystalline momentum. Let the basis states be normalized to unity. Then the diagonal elements of formulas (33) reduce to

$$
\mathcal{L}_{\sigma}=\Sigma_{m}\left|\lambda_{m, \sigma}(\mathbf{k})\right|^{2} / v_{x, m, \sigma}(\mathbf{k}), \quad \mathcal{M}_{\sigma^{\prime}}=\Sigma_{n, \sigma^{\prime}}\left|\mu_{n, \sigma^{\prime}}(\mathbf{k})\right|^{2} / v_{x, n, \sigma}(\mathbf{k})
$$

with factors independent of $\sigma$ and $\sigma^{\prime}$ omitted. Here $v_{x, m, \sigma}=\partial \varepsilon_{m, \sigma}(\mathbf{k}) / \partial k_{x}$ and $v_{x, n, \sigma^{\prime}}=\partial \varepsilon_{n, \sigma^{\prime}}(\mathbf{k}) / \partial k_{x}$ are velocity components normal to the junction plane. Their presence in these formulas follows from the restriction on $\Sigma^{\prime}$ in the basic formula (8).

To evaluate Eq. (38) for finite disorder, specialize to small $V$ and constant $\mathcal{U}$ inside $\mathcal{B}$. After evaluation of the integral in Eq. (31), it reduces to the form

$$
\Gamma_{\sigma, \sigma^{\prime}}=f_{1} \sum_{\mathbf{k}} \kappa^{2}(\mathbf{k}) e^{-2 w \kappa(\mathbf{k})} \mathcal{L}_{\sigma}(\mathbf{k}) \mathcal{M}_{\sigma^{\prime}}(\mathbf{k})
$$

where $f_{1}$ does not depend on $\sigma$ or $\sigma^{\prime}$. For large $w$, this sum weights small $k$ heavily, as mentioned above. Therefore parametrize $\mathcal{L}_{\sigma}$ and $\mathcal{M}_{\sigma^{\prime}}$ for small $k$ with the lateral 
spatial correlation scales $\left(\xi_{\sigma}, \eta_{\sigma^{\prime}}\right)$ defined by the formulas

$$
\begin{aligned}
\mathcal{L}_{\sigma}(\mathbf{k}) & =\mathcal{L}_{\sigma}(0)\left[1-\xi_{\sigma}^{2} k^{2}+\mathcal{O}\left(k^{4}\right)\right], \\
\mathcal{M}_{\sigma^{\prime}}(\mathbf{k}) & =\mathcal{M}_{\sigma^{\prime}}(0)\left[1-\eta_{\sigma^{\prime}}^{2} k^{2}+\mathcal{O}\left(k^{4}\right)\right]
\end{aligned}
$$

and approximate Eq. (29) with $\kappa \approx \kappa_{0}+\left(k^{2} / 2 \kappa_{0}\right)$ in the exponent of Eq. (31). After approximating $\sum_{\mathrm{k}}$ (over one BZ) with an infinite integral, one finds by elementary integration a result equivalent, to first order in $w^{-1}$, to

$$
\begin{aligned}
\Gamma_{\sigma, \sigma^{\prime}}(w) & \approx \Gamma_{\sigma, \sigma^{\prime}}^{(0)}(w)+\Gamma_{\sigma, \sigma^{\prime}}^{(1)}(w) \\
& \approx f_{2}(w) \mathcal{L}_{\sigma}(0)\left(1-\frac{\kappa_{0} \xi_{\sigma}^{2}}{w+\kappa_{0}^{-1}}\right) \mathcal{M}_{\sigma^{\prime}}(0)\left(1-\frac{\kappa_{0} \eta_{\sigma^{\prime}}^{2}}{w+\kappa_{0}^{-1}}\right)
\end{aligned}
$$

where once again factors independent of both $\sigma$ and $\sigma^{\prime}$ are absorbed into $f_{2}$. Thus to this approximation, $\Gamma_{\sigma, \sigma^{\prime}}$ once again has the factored form (16). (It appears that in order $w^{-2}, \Gamma_{\sigma, \sigma^{\prime}}$ does not separate this way into left- and right-dependent factors.) The corrected left polarization factor, according to Eq.(18) reduces upon expansion to

$$
\begin{aligned}
P_{\mathrm{L}} & =P_{\mathrm{L}}^{(0)}+\frac{1}{2}\left(1-P_{\mathrm{L}}^{(0) 2}\right) \frac{\kappa_{0}\left(\xi_{-}^{2}-\xi_{+}^{2}\right)}{w+\kappa_{0}^{-1}}+\ldots \quad \text { with } \\
P_{\mathrm{L}}^{(0)} & =\frac{\mathcal{L}_{+}(0)-\mathcal{L}_{-}(0)}{\mathcal{L}_{+}(0)+\mathcal{L}_{-}(0)}
\end{aligned}
$$

and similarly for $P_{\mathrm{R}}$. Thus, from given Bardeen basis functions one can obtain polarization factors, correctly to order $w^{-1}$, in a disordered electrode-barrier combination.

\section{Discussion}

Although it is valid only in the limit of weak transmission, predictions from Bardeen's tunneling theory [18] are interesting because it does not require electron momentum within the electrodes to be conserved. Our application to elastic tunneling through ordered or disordered magnetic tunneling junctions yields these new conclusions:

- In Section 3 we found that the torque at constant external voltage is generally proportional to $\sin \theta$ [Eq.(13)] . This result is a direct consequence of the single-transition nature of tunneling and the simple form of the spinor transformation (6). It contrasts with the more general angular dependence conditioned on electron structure and spin-channel resistance parameters in the case of a metallic spacer [20]. 
- In general, polarization factors do not exist in the absence of special assumptions, in agreement with previous theory [9, 12].

- In Section 4 we found that if the polarization factors are well defined, then at constant applied voltage the electric current and in-plane torque obey the relations (1), (13), and (20). These similar relations are inter-connected by the presence of the common factor $J_{0}(V)$ which we do not attempt to calculate. The dimensionless coefficients in these relations are expressed in terms of the polarizations by $\tau_{\mathrm{R}}=P_{\mathrm{L}}, \tau_{\mathrm{L}}=P_{\mathrm{R}}, \iota=P_{\mathrm{L}} P_{\mathrm{R}}$, implying $\iota=\tau_{\mathrm{L}} \tau_{\mathrm{R}}$. In particular, these general relations are satisfied by the special results of a direct solution of the Schroedinger equation for the toy model of parabolic bands and ideal rectangular potential barrier [1].

- Experimentally, TMR is known to usually diminish with increasing external voltage $V[9,10]$. In Section 5 we considered that it is the polarizing factor of the collector electrode which decreases more strongly with $V$, resulting in the unsymmetric schematic pattern of voltage dependence of torque indicated in Fig. 3. This lack of symmetry due to the relations $\tau_{\mathrm{R}}=P_{\mathrm{L}}$ and $\tau_{\mathrm{L}}=$ $P_{\mathrm{R}}$ implies that the threshold voltage for initiation of dynamic excitation will be increasingly asymmetric at the higher values $(>100 \mathrm{mV})$ likely needed for writing in memory. Cases may well arise in which voltage-driven switching works in only one direction. For selected experimental junctions, switching is observed at a voltage sufficiently high for TMR to become negligible [7]. Our Fig. 3 indicates how this may happen for switching in but one direction, from AP to P. However, our theory would not explain any symmetric persistence of switching at voltages great enough to destroy TMR, if this is observed.

- Our approach to the validation of polarization factors complements previous studies which accounted for atomic disorder in the barrier assuming electrode states with well-defined crystalline momentum $[15,16,17]$. We assume that the barrier is thick and includes an ideal crystalline or vacuum middle region of thickness $w$ as in Fig. 4. Then a newly derived polarization factor, given by Eq. (43), is valid to first order in $w^{-1}$ even in the presence of disorder in the electrodes and interfaces sufficient to destroy the conservation of lateral crystalline momentum throughout the electrode and interface regions. The key basis-state weight factors (36) and (37) are more general than the conventional local state density.

- Our conclusion that the validity of polarization factors increases with increasing $w$ tends to undermine our predictions of voltage asymmetry of torque shown 
schematically in Fig. 3. For, experimental spin-transfer effects such as switching will require very thin barriers, making the separability condition assumed in Fig. 3 less valid. Previous proposals $[12,17]$ that validity of polarization factors is attributable to unique defect states or amorphicity in the barrier are more promising in this respect.

- Belashenko and co-authors [17] find that certain first-principle TMR computations for realistic barrier thickness may be poorly approximated by proportionality to $e^{-\kappa w}$. This casts additional doubt on the applicability of the ideal middle to the very thin junctions needed for spin-momentum transfer experiments. However, our conclusions from this model may bear significantly on magneto-resistance experiments carried out with greater thickness, as suggested below.

- Our parametrized expression (43) for dependence of tunnel polarization on ideal-middle thickness $w$ is without precedent. A strong dependence is expected from certain compositions, like $\mathrm{Co}, \mathrm{Ni}$, and certain alloys, such as FeCo, lying on the negative-slope region of the Slater-Pauling curve [34]; for, their strong contrast between heavily 4sp-weighted density of majority-spin and heavily $3 \mathrm{~d}$-weighted density of minority-spin bands may be reflected in strongly contrasting magnitudes of left lateral autocorellation scales $\xi_{+}$and $\xi_{-}$. Theoretical estimation of the left polarization factor will require prior first-principle computation of the Bardeen basis functions $\psi_{p, \sigma}$ for the disordered electrodebarrier system. From these, one must invert the series (27) to evaluate the diagonal elements of the Fourier coefficients $\lambda_{p, \sigma}$. Then Taylor expansion of the diagonal element in the first Eq. (33) for substitution into the first Eq. (40) provides the coefficients $\mathcal{L}_{ \pm}(0)$ and $\xi_{ \pm}$. These parameters must then be substituted into Eqs. (43) to obtain the left polarization factor.

- In fact, experimental junctions having composition $\mathrm{Fe} / \mathrm{Al}_{2} \mathrm{O}_{3} / \mathrm{FeCo}$ show dependence of TMR on barrier thickness [10] at $\mathrm{T}=2 \mathrm{~K}$ where our assumption of elastic tunneling should be valid. A monotonic dependence on thickness, expected from Eq. (42), is observed for two crystallographic orientations on single-crystal Fe, but not on the third. Although the (say) right electrode (FeCo) lies on the negative-slope side, the left electrode (Fe) lies on the positiveslope side of the Slater-Pauling curve where high $3 \mathrm{~d}$ density exists for both signs of spin so that there may be little difference between $\xi_{+}$and $\xi_{-}$. Junctions with both electrodes taken from the negative-slope side may yield a more pronounced thickness dependence of TMR on barrier thickness according to the 
present theory.

Acknowledgments. The author is grateful to G. Mathon for a related preprint, and for helpful discussions with W. Butler, E. Tsymbal, K. Belashchenko, M. Stiles, Y. Bazaliy, J. Sun, S. S. P. Parkin, P. Nguyen, G. Fuchs, D. Worledge, and P. Visscher.

\section{References}

[1] J. C. Slonczewski, Phys. Rev. B, 39, 6995 (1989).

[2] S. I. Kiselev et al, Nature , 425, 380 (2003).

[3] W. H. Rippard, M. R. Pufall, S. Kaka, S. E. Russek, and T. J. Silva, Phys. Rev. Lett. 92, 027201(2004).

[4] J. S. Moodera, L. R. Kinder, T. M. Wong, and R. Meservey, Phys. Rev. Lett. 74, 3273 (1995).

[5] S. S. P. Parkin, in Spin Dependent Transport in Magnetic Nanostructures (Taylor \& Francis, 2002), p. 237.

[6] Y. Huai, F. Albert, P. Nguyen, M. Pakala, and T. Valet, Appl. Phys. Lett. 84, 3118 (2004).

[7] G. D. Fuchs, N. C. Emley, I. N. Krivorotov, P. M. Braganca, E. M. Ryan, S. I. Kiselev, J. C. Sankey, D. C. Ralph, R. A. Buhrman, and J. A. Katine, Appl. Phys. Lett. 85, 1205 (2004).

[8] J. Slonczewski, Frontiers in Magnetism 99. Stockholm, 12-15 August 1999, arXiv: cond-mat/0205055.

[9] J. S. Moodera and G. Mathon, J. Mag. Mag. Mats. 200, 248 (1999).

[10] T. Miyazaki, Chapter 3 in Spin Dependent Transport in Magnetic Nanostructures, edited by S. Maekawa and T. Shinjo (Taylor \& Francis, 2002).

[11] S. Maekawa, S. Takahashi, and H. Imamura, Chapter 4 in Spin Dependent Transport in Magnetic Nanostructures (Taylor \& Francis, 2002).

[12] E. Y. Tsymbal, O. N. Mryasov, and P. R. LeClair, J. Phys.: Condens. Matter 15, R109 (2003).

[13] R. Meservey and P. M. Tedrow, Physics Reports 238, 173 (1994).

[14] M. Julliere, Phys. Lett. 54A, 225 (1975). 
[15] E. Y. Tsymbal and D. G. Pettifor, Phys. Rev. B 58, 432 (1998).

[16] J. Mathon and A. Umerski, Phys. Rev. B 60, 1117(1999).

[17] K. D. Belashchenko, E. Y. Tsymbal, M. van Schilfgaarde, D. A. Stewart, I. I. Oleynik, and S. S. Jaswal, Phys. Rev. B 69, 174408 (2004).

[18] J. Bardeen, Phys. Rev. Letts. 6, 57 (1961).

[19] C. B. Duke, Tunneling in Solids, (Academic Press, New York, 1969), Sec. 18a, Eqs. (8.11-8.14)

[20] J. Slonczewski, J. Magn. Magn. Mater. 247, 324 (2002).

[21] L. Berger, Phys. Rev. B 54, 9353 (1996).

[22] Y. B. Bazaliy, B. A. Jones, and S.-C. Zhang, Phys. Rev. B 57, R3213 (1998).

[23] J. Slonczewski, J. Magn. Magn. Mats. 195, L261 (1999).

[24] M. D. Stiles and A. Zangwill, Phys. Rev. B 66, 014407 (2002).

[25] F. J. Albert, N. C. Emley, E. B. Myers, D. C. Ralph, and R. A. Buhrman,Phys. Rev. Lett. 89, 226802 (2002).

[26] D. M. Edwards, F. Federici, J. Mathon, and A. Umerski, preprint.

[27] J. Bass and W. P. Pratt, Jr., J. Magn. Magn.Mats. 200, 274 (1999).

[28] J. Kübler, Theory of Itinerant Electron Magnetism (Oxford Science Publications, 2000).

[29] T. Valet and A. Fert, Phys. Rev. B 48, 7099 (1993).

[30] Eq. (18.34) in Ref. [19].

[31] N. Tezuka and T. Miyazaki, J. Magn. and Magn. Mats. 177-181, 1283 (1998).

[32] Fei-fei Li, Zheng-zhong Li, Ming-wen Xiao, Jun Du, Wang Xu, An Hu, and John Q. Xiao, J. Appl. Phys. 957243 (2004).

[33] See, for example, A. C. Smith, J. F. Janak, and R. S. Adler, Electronic Conduction in Solids (McGraw-Hill 1967) p. 125.

[34] Ref. [28], p. 234. 